\title{
Understanding the Role of Electronic Effect in CO on Pt-Sn alloy Surface via Band Structure Measurements
}

AUTHOR NAMES Jongkeun Jung ${ }^{1,2}$, Sungwoo Kang ${ }^{3}$, Laurent Nicolaï ${ }^{4}$, Jisook Hong 5 , Ján Minár ${ }^{6}$, Inkyung Song ${ }^{1,2}$, Wonshik Kyung ${ }^{1,2}$, Soohyun $\mathrm{Cho}^{7}$, Beomseo Kim ${ }^{1,2}$, Jonathan D. Denlinger ${ }^{8}$, Francisco José Cadete Santos Aires ${ }^{9,10}$, Eric Ehret ${ }^{9}$, Philip Ross ${ }^{11}$, Jihoon Shim ${ }^{12}$, Slavomir Nemšák ${ }^{8}$, Doyoung Noh ${ }^{13,14}$, Seungwu $\operatorname{Han}^{3 *}$, Changyoung Kim ${ }^{1,2 *}$, Bongjin Simon Mun ${ }^{13,14 *}$

\section{AUTHOR ADDRESS}

${ }^{1}$ Center for Correlated Electron Systems, Institute for Basic Science 1 Gwanak-ro, Gwanak-gu, Seoul, 08826, Republic of Korea

${ }^{2}$ Department of Physics and Astronomy, Seoul National University 1 Gwanak-ro, Gwanak-gu, Seoul, 08826, Republic of Korea

${ }^{3}$ Department of Materials Science and Engineering, Seoul National University, 1 Gwanak-ro, Gwanak-gu, Seoul, 08826, Republic of Korea

${ }^{4}$ New Technologies Research Centre (NTC), University of West Bohemia, Univerzitni 8/2732 301 00, Plzen, Czech Republic

${ }^{5}$ The Molecular Foundary, Lawrence Berkeley National Laboratory, 1 Cyclotron Rd, Berkeley, California, 94720, USA

${ }^{6}$ New Technologies Research Centre (NTC), University of West Bohemia, Univerzitni 8/2732 301 00, Plzen, Czech Republic

${ }^{7}$ Shanghai Institute of Microsystem and Information Technology (SIMIT), Chinese Academy of Sciences, 865 Changning Rd, Shanghai, 200050, People's Republic of China

${ }^{8}$ Advanced Light Source, Lawrence Berkeley National Laboratory, 1 Cyclotron Rd, Berkeley, California, 94720, USA

${ }^{9}$ Université Claude Bernard Lyon 1, Université de Lyon, CNRS - UMR 5256, IRCELYON 2, Avenue Albert Einstein, 69626 Villeurbanne Cedex, France.

${ }^{10}$ National Research Tomsk State University, Laboratory for Catalytic Research. 36, Lenin Avenue, 634050 Tomsk, Russian Federation.

${ }^{11}$ Materials Science Division, Lawrence Berkeley National Laboratory, 1 Cyclotron Rd, Berkeley, California, 94720, USA

${ }^{12}$ Department of Chemistry, Pohang University of Science and Technology, San 31, Hyojadong, Namgu, Pohang, 37673, Republic of Korea

${ }^{13}$ Department of Physics and Photon Science, Gwangju Institute of Science and Technology, 123 Cheomdangwagiro, Buk-gu, Gwangju, Republic of Korea.

${ }^{14}$ Center for Advanced X-ray Science, Gwangju Institute of Science and Technology, 123 Cheomdan-gwagiro, Buk-gu, Gwangju, Republic of Korea. 


\section{Experimental details}

\section{Experimental details}

Clean $\mathrm{Pt}(111)$ surface is obtained from repeated cycle of $\mathrm{Ar}^{+}$ion sputtering $\left(1.5 \mathrm{keV}, \mathrm{I}_{\text {emission current }}=6 \mathrm{~mA}, \mathrm{P}(\mathrm{Ar})\right.$ $=3 \times 10^{-6}$ Torr for $\left.10 \mathrm{mins}\right)$ and annealing $(\mathrm{T}=1000 \mathrm{~K}$ for $2 \mathrm{mins})$. The cleaning cycle was repeated until no carbon signal is observed. The surface cleanness was checked with Auger electron spectroscopy (AES). All the LEED and AES data were taken with a SPECS ErLEED 3000-D. The residual gases in a high vacuum chamber were checked with a mass spectrometer (SRS RGA 100), and the partial pressure of $\mathrm{H}_{2}, \mathrm{H}_{2} \mathrm{O}, \mathrm{CO}$ and $\mathrm{CO}_{2}$ gases were $5.9 \times 10^{-10}, 4.0 \times 10^{-11}, 2.8 \times 10^{-11}$ and $9.1 \times 10^{-12}$ Torr, respectively. No sign of carbon was observed from AES prior to the ARPES measurement. The measurement time for ARPES was 1 hour for a specific band, and 2 hours for full mapping. Under the current chamber pressure condition, with normal molecular sticking coefficients, more than 20 hours is needed to have $1.0 \mathrm{ML}$ coverage of impurity molecules $\left(\mathrm{H}_{2}, \mathrm{H}_{2} \mathrm{O}\right.$, and $\left.\mathrm{CO}_{2}\right)$ on $\mathrm{Pt}(111)$ at $100 \mathrm{~K}$. During the measurement of ARPES, no significant changes were found.
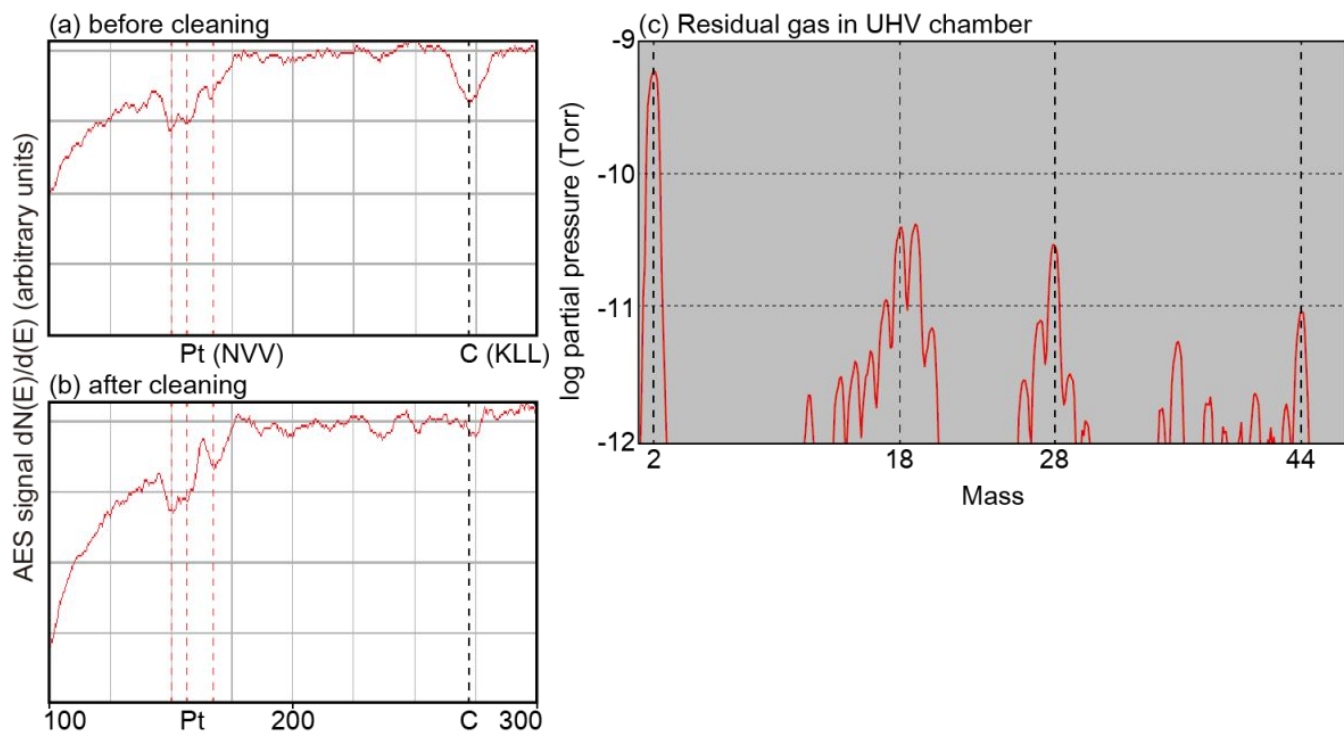

Figure S1. AES data of $\mathrm{Pt}(111)$ (a) before and (b) after cleaning. (c) The partial pressure of residual gas measured with mass spectrometer in UHV chamber during ARPES measurement. 
(a) Pt

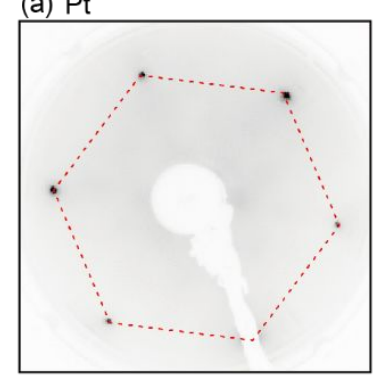

(d) $\sim 0.25 \mathrm{ML} \mathrm{CO} / \mathrm{Pt}$

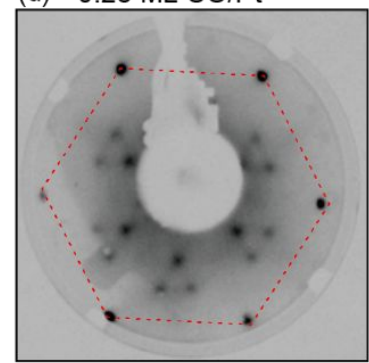

(b) Pt-Sn $\sqrt{3} x \sqrt{3}$

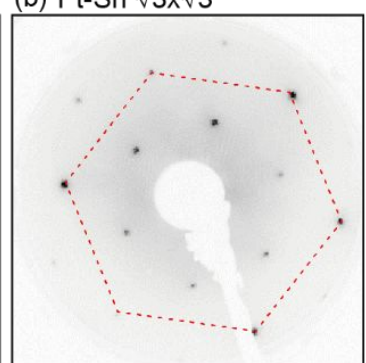

(e) $\sim 0.5 \mathrm{ML} \mathrm{CO} / \mathrm{Pt}$

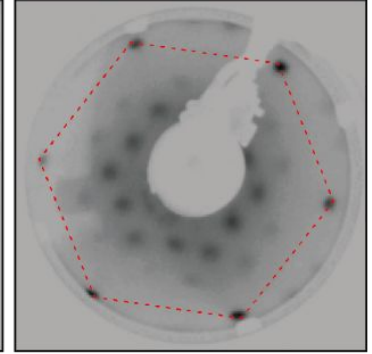

(c) Pt-Sn $2 \times 2$

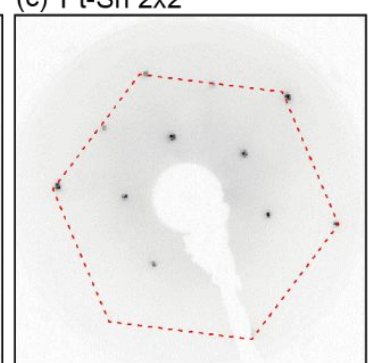

(f) $\sim 0.5 \mathrm{ML} \mathrm{CO} / \mathrm{Pt}-\mathrm{Sn} 2 \times 2$

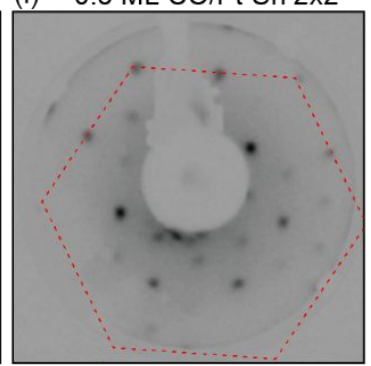

Figure S2. LEED patterns of $\mathrm{Pt}(111), \mathrm{Pt}-\mathrm{Sn} / \mathrm{Pt}(111) \sqrt{3} \times \sqrt{3}$ surface alloy, and $\mathrm{Pt}-\mathrm{Sn} / \mathrm{Pt}(111) 2 \times 2$ surface alloy, and changes in the surface arrangement by $\mathrm{CO}$ adsorption: (a) $\operatorname{Pt}(111)$, (b) $\mathrm{Pt}-\operatorname{Sn} / \mathrm{Pt}(111) \sqrt{3} \times \sqrt{3}$, (c) $\mathrm{Pt}$ $\mathrm{Sn} / \mathrm{Pt}(111) 2 \times 2$, (d) 0.25 ML CO/Pt, (e) $\sim 0.5 \mathrm{ML} \mathrm{CO} / \mathrm{Pt}$, and (f) 0.5 ML CO/Pt-Sn/Pt(111) $2 \times 2$. Dotted lines indicate the Brillouin zone boundary.

Two different $\mathrm{Pt}-\mathrm{Sn} / \mathrm{Pt}(111)$ surface alloys, $\sqrt{3} \times \sqrt{3}$ and $2 \times 2$, are prepared by Sn deposition and annealing on a Pt single crystal surface at 800 and $1,100 \mathrm{~K}$, respectively. The surface arrangements of $\mathrm{Pt}(111)$ and the Pt$\mathrm{Sn} / \mathrm{Pt}(111)$ surface alloy are confirmed with LEED. ${ }^{1,2} \mathrm{CO}$ is dosed on the sample surface using gas pipe close from the sample to keep the base pressure low. While changes in LEED patterns are observed between Pt and the $\mathrm{Pt}-\mathrm{Sn} / \mathrm{Pt}(111) 2 \times 2$ surface alloy with $\mathrm{CO}$ adsorption no such changes are observed with the $\mathrm{Pt}-\mathrm{Sn} / \mathrm{Pt}(111) \sqrt{3} \times$ $\sqrt{3}$ surface; this is because $\mathrm{CO}$ molecules do not form an well-ordered arrangement on the $\sqrt{3} \times \sqrt{3}$ surface due to the increased proportion of $\mathrm{Sn} .{ }^{3} \mathrm{CO}$ adsorbates form ordered adlayers on $\mathrm{Pt}$ and $\mathrm{Pt}-\mathrm{Sn} / \mathrm{Pt}(111) 2 \times 2$ surface alloy. Two different adlayer configurations for $\mathrm{CO} / \mathrm{Pt}$ and $\mathrm{CO} / \mathrm{Pt}-\mathrm{Sn} / \mathrm{Pt}(111) 2 \times 2$ surface alloy are confirmed by comparing LEED patterns. ${ }^{4,5}$ At $0.25 \mathrm{ML}$ coverage on $\mathrm{Pt}(111)$, CO sits on the top site and forms a $\sqrt{3} \times \sqrt{3}$ superstructure. At $0.5 \mathrm{ML}, \mathrm{CO}$ sits on both top and bridge sites with $\mathrm{c}(4 \times 2)$ structure. In the case of of Pt-Sn 2 $\times 2$ surface alloy surface, no superstructure could be identified at $0.25 \mathrm{ML}$, consistent with a previous report ${ }^{3}$, while $2 \sqrt{3} \times 2 \sqrt{3}$ superstructure was observed at $0.5 \mathrm{ML}$ coverage of $\mathrm{CO}$ without any mixed phase. The real space model for $\mathrm{CO} / \mathrm{Pt}(111)$ and $\mathrm{CO} / \mathrm{Pt}-\mathrm{Sn} / \mathrm{Pt}(111) 2 \times 2$ surface alloy can be found in references. ${ }^{3,5}$ During each measurement of ARPES, we checked the surface ordering with LEED, which exhibits the presence of single ordered phase. Previously, $\mathrm{CO}$ binding energies on $\mathrm{Pt}(111)^{6}$ and $\mathrm{Pt}_{3} \mathrm{Sn}(111)^{7}$ bulk alloy surfaces were calculated using DFT. At low coverage (1/9 ML), the most stable adsorption site of CO on $\mathrm{Pt}(111)$ was the top site with 1.52 $\mathrm{eV}$ binding energy. At $0.5 \mathrm{ML}$ coverage, 2 top and 2 bridge site $\mathrm{CO}$ atoms on the $\mathrm{c}(4 \times 2)$ unit cell are most stable

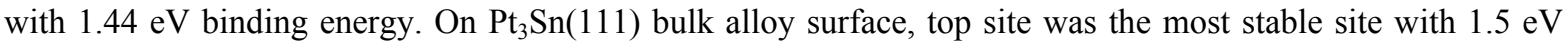
binding energy for $0.25 \mathrm{ML}$ CO coverage. For $0.5 \mathrm{ML}$ CO coverage, hollow site becomes more stable with 1.27 eV binding energy. 
The atomic model for DFT calculations.

(a) $0.33 \mathrm{ML} \mathrm{CO} / \mathrm{Pt}$
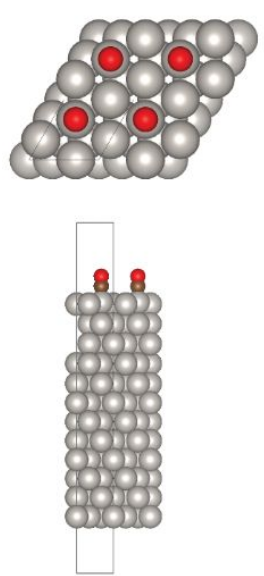

(b) $0.5 \mathrm{ML} \mathrm{CO} / \mathrm{Pt}$
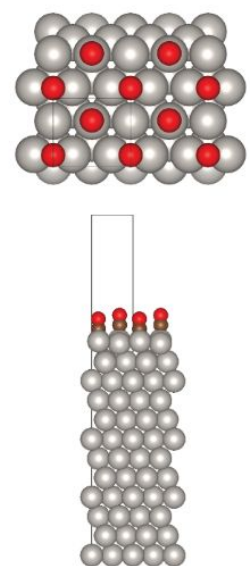

(c) Pt-Sn/Pt
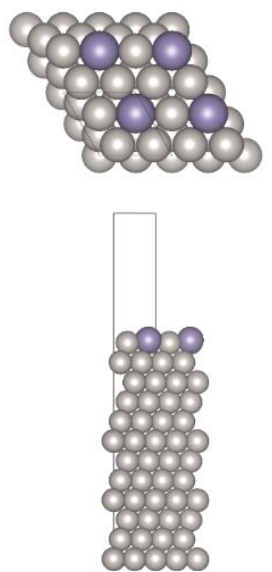

(d) $0.5 \mathrm{ML} \mathrm{CO} / \mathrm{Pt}-\mathrm{Sn} / \mathrm{Pt}$
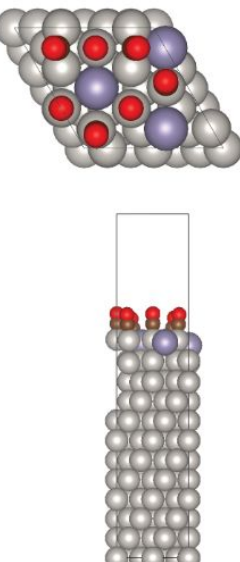

Figure S3. The atomic model of (a) $0.33 \mathrm{ML} \mathrm{CO} / \mathrm{Pt}$, (b) $0.5 \mathrm{ML} \mathrm{CO} / \mathrm{Pt}$, (c) Pt-Sn/Pt, and (d) CO/Pt-Sn/Pt. The gray, purple, black, and red atoms represent $\mathrm{Pt}, \mathrm{Sn}, \mathrm{C}$, and $\mathrm{O}$, respectively. The $\mathrm{CO}$ distribution of $0.5 \mathrm{ML} \mathrm{CO} / \mathrm{Pt}-$ $\mathrm{Sn} / \mathrm{Pt}$ are employed from reference. ${ }^{3}$ In actual comparison with the experimental data in Fig. 3 , the atomic model of $0.33 \mathrm{ML} \mathrm{CO} / \mathrm{Pt}$ is compared with experimental $0.25 \mathrm{ML} \mathrm{CO} / \mathrm{Pt}$ system since both show a $\sqrt{3} \times \sqrt{3} \mathrm{LEED}$ pattern (Figure S2) while our 0.25 ML model has a different structure.

Table S1. Calculated average adsorption energies of CO.

\begin{tabular}{ll}
\hline \hline Material & $\mathrm{E}(\mathrm{eV})$ \\
\hline $0.5 \mathrm{CO} \mathrm{ML} / \mathrm{Pt}$ & -1.38 \\
$0.58 \mathrm{CO} \mathrm{ML} / \mathrm{Pt}-\mathrm{Sn} / \mathrm{Pt}$ & -0.93 \\
\hline \hline
\end{tabular}

ARPES simulation for Pt and Pt-Sn/Pt(111) $2 \times 2$ surface alloy.
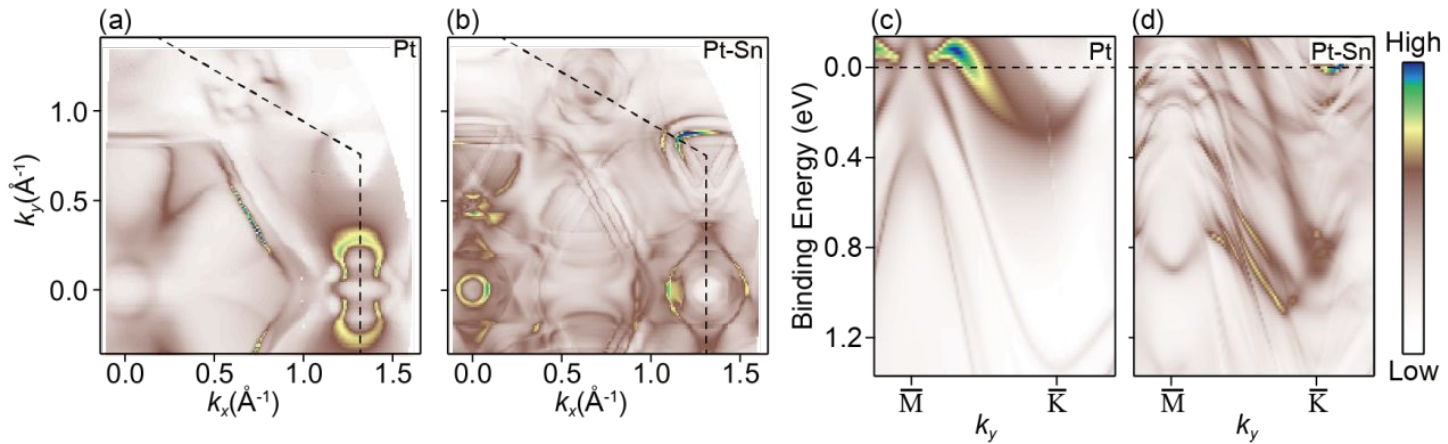

Figure S4. (a) Pt, and (b) Pt-Sn/Pt(111) $2 \times 2$ surface alloy Fermi surfaces and band structures of (c) Pt, and (d) $\mathrm{Pt}-\mathrm{Sn} / \mathrm{Pt}(111) 2 \times 2$ surface alloy near the M point obtained with ARPES simulation.

The DFT calculations for ARPES simulation are based on a fully relativistic multiple scattering approach (Korringa-Kohn-Rostoker (KKR) method), as implemented in the SPR-KKR package. ${ }^{8}$ As the first step in these theoretical investigations, we performed self-consistent ground state calculations for 2D semi-infinite $\operatorname{Pt}(111)$ and reconstructed Pt-St/Pt(111) $2 \times 2$ surfaces. The self-consistent results served as an input for the spectroscopic investigations. ARPES calculations are performed in the framework of the fully relativistic one-step model of photoemission using experimental geometry and photon energy. ${ }^{9}$ Thus, the theory accounts for effects induced by light polarization, matrix-element effects, final state effects, and surface effects. 

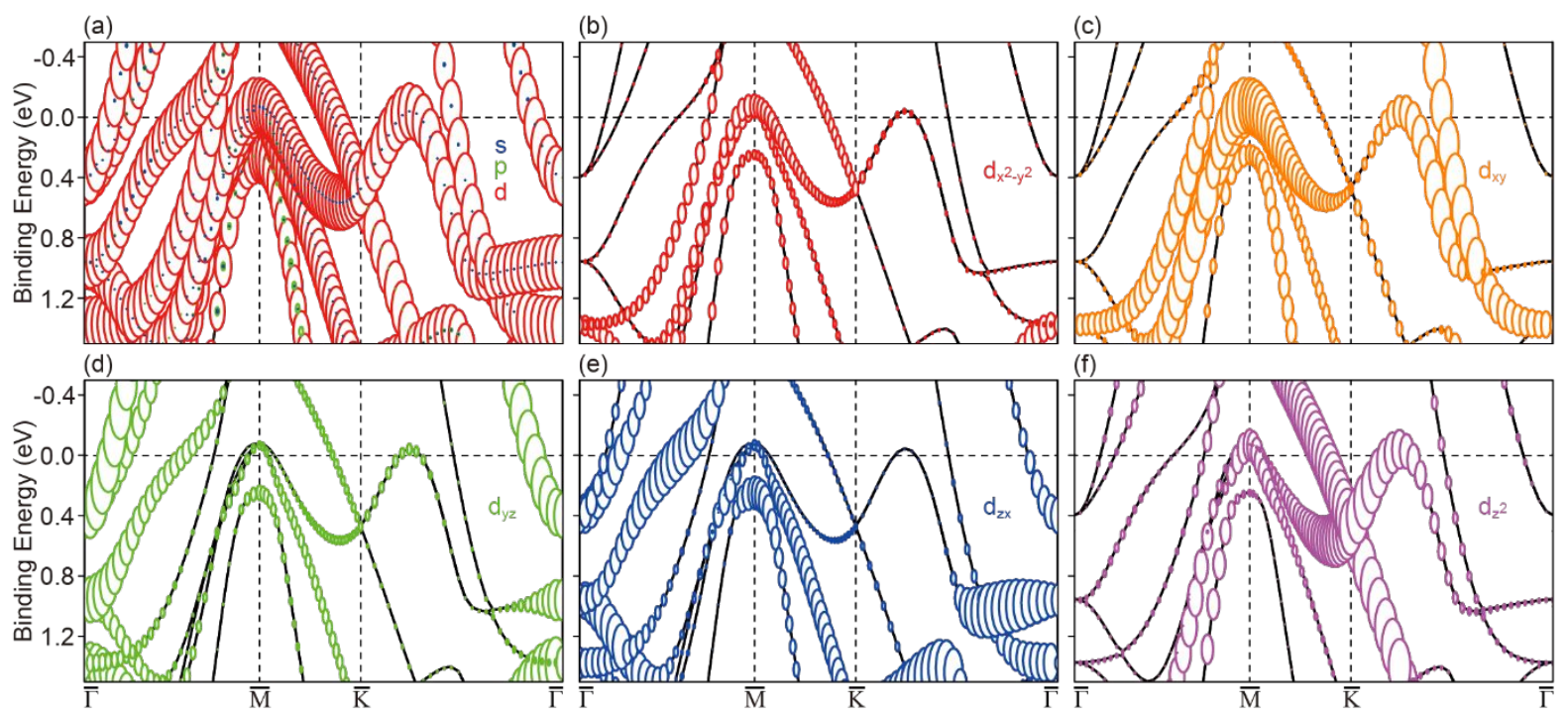

Figure S5. Orbital character calculations of Pt(111). Orbital contributions of (a) s, p, d, (b) $d_{x 2-y 2}$, (c) $d_{x y}$, (d) $d_{y z}$, (e) $d_{z x}$, and (f) $d_{z 2}$ orbitals are indicated by circles proportional to the contribution.

Figure S5 shows the orbital contribution of $\mathrm{Pt}(111)$ bands near the Fermi level. The orbital contribution is indicated by the size of the circle. The bands near the Fermi level of $\operatorname{Pt}(111)$ are mostly $d$-bands (Figure S3a) and the detailed orbital character of these $d$-bands are shown in Figures S3b-f. Figures S3b-f shows the $d_{\mathrm{x} 2-\mathrm{y} 2}, \mathrm{~d}_{\mathrm{xy}}, \mathrm{d}_{\mathrm{yz}}$, $\mathrm{d}_{\mathrm{zx}}$, and $\mathrm{d}_{\mathrm{z} 2}$ orbital contributions, respectively. DFT calculations are performed with the Vienna ab initio simulation package (VASP) based on the frozen-core full-potential projector augmented-wave (PAW) method, ${ }^{10,11}$ under the generalized gradient approximation (GGA) of Perdew-Burke-Ernzerhof (PBE) as an exchange correlation functional. ${ }^{12} \mathrm{We}$ optimized the cell parameters of fcc Pt until the force became less than 10-9 $\mathrm{eV} / \AA \AA$ using a $7 \times 7 \times 7 \Gamma$-centered K-points mesh. This resulted in a cell parameter of $2.81296 \AA$ for a primitive unit cell. Based on this, we constructed a hexagonal supercell of fcc Pt featuring ABC stacking of three Pt triangular lattices. The electronic structure of the Pt supercell, which we will refer to hereafter as $\operatorname{Pt}(111)$, is relaxed to include spin-orbit coupling with a threshold of $10^{-3} \mathrm{eV}$. 


\section{Orbital character change of hexagonal band due to the $\mathrm{CO}$ adsorption.}
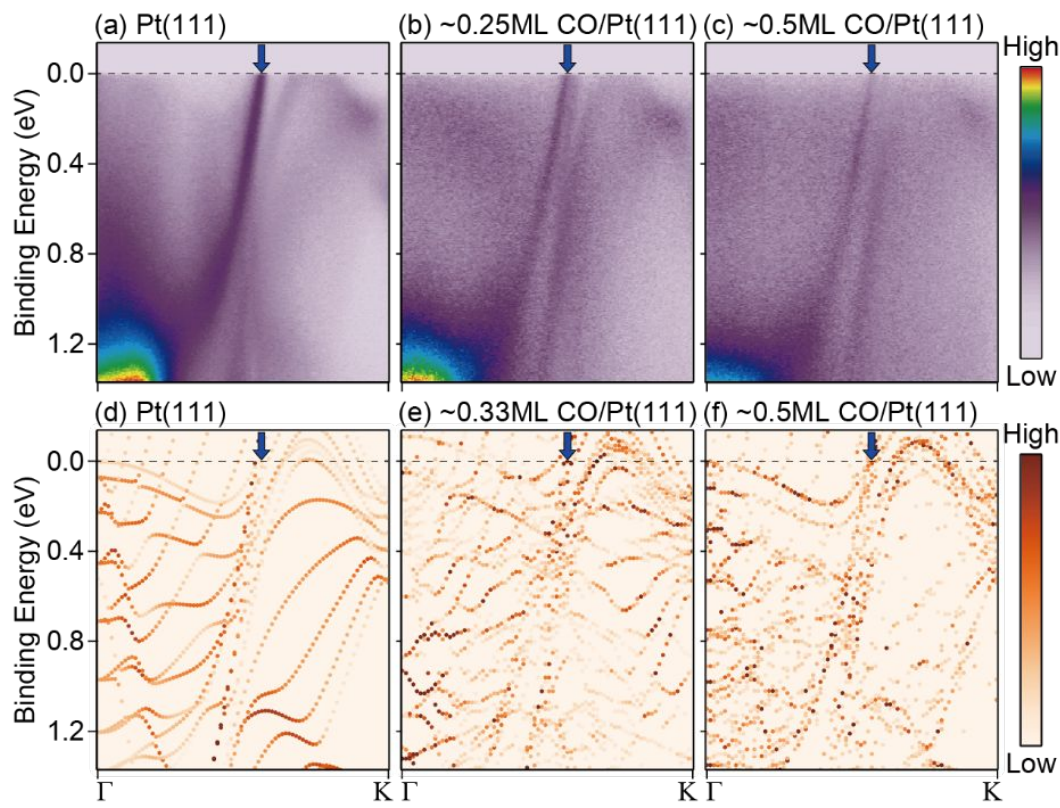

(e) $0.33 \mathrm{ML} \mathrm{CO} / \mathrm{Pt}(111)$ (f) $0.5 \mathrm{ML} \mathrm{CO} / \mathrm{Pt}(111)$

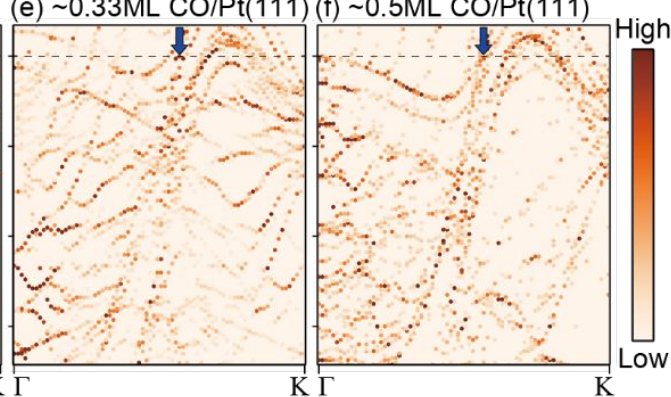

Figure S6. Band structures of (a) $\mathrm{Pt}(111)$, (b) $\sim 0.25 \mathrm{ML} \mathrm{CO} / \mathrm{Pt}(111)$, and (c) $\sim 0.5 \mathrm{ML} \mathrm{CO} / \mathrm{Pt}(111)$ along the $\Gamma \mathrm{K}$ direction, and DFT calculation of (d) $\mathrm{Pt}(111)$, (e) 0.33 ML CO/Pt(111), and (f) $\sim 0.5 \mathrm{ML} \mathrm{CO} / \mathrm{Pt}(111)$ along the $\Gamma \mathrm{K}$ direction. The hexagonal bands are marked with blue arrows.

Figure S6 shows the band structure of $\mathrm{Pt}(111)$ and $\mathrm{CO}$ adsorbed Pt surfaces along the $\Gamma \mathrm{K}$ direction and the DFT calculated band structure. Although it is not easy to recognize band splitting at the DFT calculation, the hexagonal bands become broader due to the $\mathrm{CO}$ adsorption. The orbital character calculation of the hexagonal band (Table $\mathrm{S} 1$ ) supports our intuition of surface-bulk splitting at $\mathrm{CO} / \mathrm{Pt}(111)$. The $\mathrm{Pt}(111)$ hexagonal band is mostly made of in-plane orbital band. This in-plane orbitals have weak interaction between the layer; and cause surface-bulk splitting when $\mathrm{CO}$ is adsorbed on the surface. The out-of-plane orbital contribution, especially $\mathrm{d}_{\mathrm{yz}}+\mathrm{d}_{\mathrm{xz}}$ contribution of $\mathrm{Pt}(111)$ hexagonal band increases as $\mathrm{CO}$ adsorb. This orbital contribution change shows similar trend with the orbital contribution change of $\mathrm{M}$ point band (Table 1) and shows the electronic band structure change near Fermi level during $\mathrm{CO}$ adsorption comes from the back-donation $\pi$-bonding states. Calculation details for the band structure and orbital characters are written in the main text.

Table S2. Calculated orbital character change of hexagonal band. Calculated orbital contribution for the hexagonal band of Pt during $\mathrm{CO}$ adsorption on Figures S4a-c.

\begin{tabular}{lccc}
\hline \hline Material & $\mathrm{d}_{\mathrm{z} 2}$ contribution & $\mathrm{d}_{\mathrm{yz}}+\mathrm{d}_{\mathrm{xz}}$ contribution & $\mathrm{d}_{\mathrm{xy}}+\mathrm{d}_{\mathrm{x} 2-\mathrm{y} 2}$ contribution \\
\hline $\mathrm{Pt}$ & 0.111 & 0.086 & 0.668 \\
$0.33 \mathrm{ML} \mathrm{CO} / \mathrm{Pt}$ & 0.159 & 0.114 & 0.612 \\
$0.5 \mathrm{ML} \mathrm{CO} / \mathrm{Pt}$ & 0.145 & 0.236 & 0.524 \\
\hline \hline
\end{tabular}


The second derivative of CO-induced energy band shifts of Pt and Pt-Sn/Pt(111) alloy
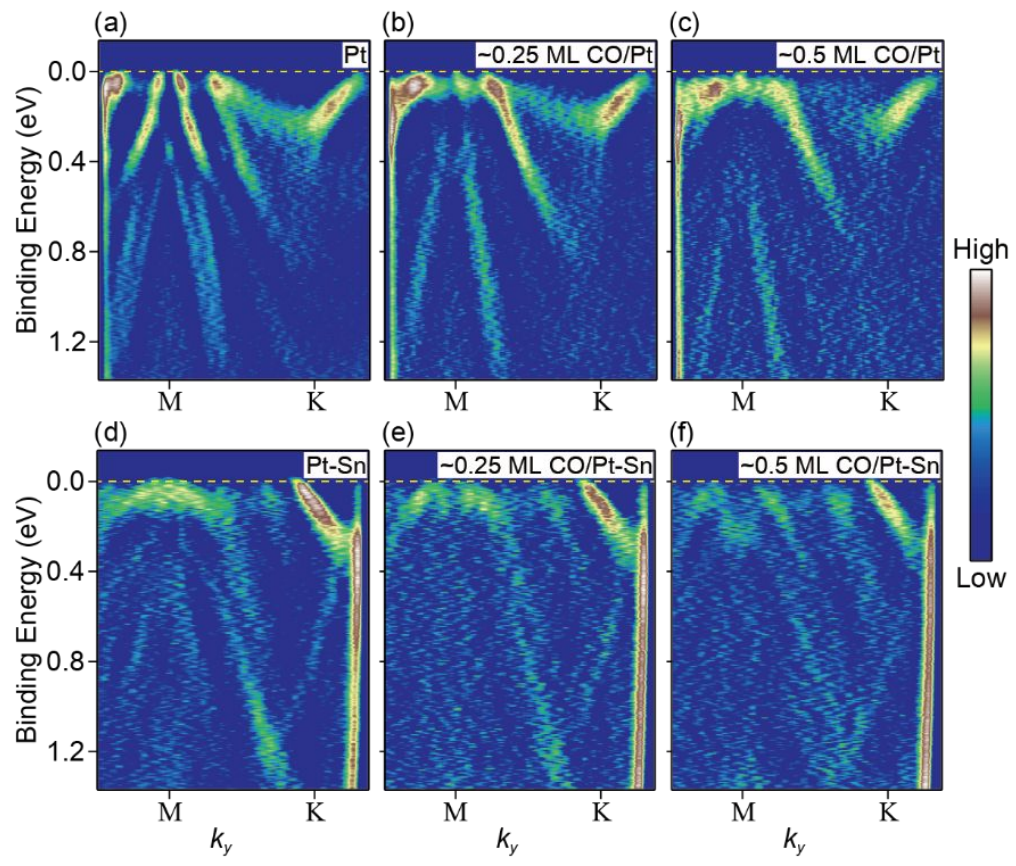

Figure S7. The second derivatives of CO-induced energy band shifts are shown at the M point of (a) $\mathrm{Pt}(111),(\mathrm{b})$ $\sim 0.25 \mathrm{ML} \mathrm{CO} / \mathrm{Pt}(111)$, (c) 0.5 ML CO/Pt(111), (d) Pt-Sn/Pt(111) surface alloy, (e) 0.25 ML CO/Pt-Sn/Pt(111) surface alloy, and (f) $\sim 0.5 \mathrm{ML} \mathrm{CO} / \mathrm{Pt}-\mathrm{Sn} / \mathrm{Pt}(111)$ surface alloy.

Figure S7 shows an intensity plot of the second derivative of Figure 3. The second derivative clearly shows the energy shifts of specific bands, indicated by yellow dotted lines in Figure 3. 


\section{Reference}

1. C. Xu, \& B. E. Koel, A LEED, TPD and HREELS Investigation of NO Adsorption on Sn/Pt(111) Surface Alloys. Surf. Sci. 1994, 310, 198-208.

2. W. C. A. N. Ceelen, A. W. Denier van der Gon, M. A. Reijme, H. H. Brongersma, I. Spolveri, A. Atrei, \& U. Bardi, Domain structure, Segregation and Morphology of the $\mathrm{Pt}_{3} \mathrm{Sn}(111)$ Surface. Surf. Sci. 1998, 406, 264-278.

3. M. T. Paffet, S. C. Gebhard, R. G. Windham, \& B. E. Koel, Chemisorption of Carbon Monoxide, Hydrogen, and Oxygen on Ordered Tin/Platinum (111) Surface Alloys. J. Phys. Chem. 1990, 94, 68316839.

4. H. Steininger, S. Lehwald, \& H. Ibach, On the Adsorption of CO on Pt(111). Surf. Sci. 1982, 123, 264282.

5. H. Hopster, \& H. Ibach, Adsorption of CO on Pt(111) and Pt 6(111)X(111) Studied by High Resolution Electron Energy Loss Spectroscopy and Thermal Desorption Spectroscopy. Surf. Sci. 1978, 77, 109-117.

6. G. K. Kalhara Gunasooriya, \& M. Saeys, CO Adsorption Site Preference on Platinum: Charge is the Essence. ACS Catal. 2018, 8(5), 3770-3774.

7. C. Dupont, D. Loffreda, F. Delbecq, \& Y. Jugnet, Vibrational Study of CO Chemisorption on the Pt3Sn (111)-(2× 2) Surface. J. Phys. Chem. C 2007, 111, 8524-8531.

8. H. Ebert, D. Ködderitzsch, \& J. Minár, Calculating Condensed Matter Properties Using the KKR-Green's Function Method-Recent Developments and Applications. Rep. Prog. Phys. 2011, 74, 096501.

9. J. Braun, J. Minár, \& H. Ebert, Correlation, Temperature and Disorder: Recent Developments in the OneStep Description of Angle-Resolved Photoemission. Phys. Rep. 2018, 740, 1-34.

10. G. Kresse, \& J. Furthmüller, Efficient Iterative Schemes for Ab Initio Total-Energy Calculations Using a Plane-Wave Basis Set. Phys. Rev. B 1996, 54, 11169.

11. G. Kresse, \& J. Furthmüller, Efficiency of Ab-Initio Total Energy Calculations for Metals and Semiconductors Using a Plane-Wave Basis Set. Comput. Mater. Sci. 1996, 6, 15-50.

12. J. P. Perdew, K. Burke, \& M. Ernzerhof, Generalized Gradient Approximation Made Simple. Phys. Rev. Lett. 1996, 77, 3865. 